\title{
Planet formation in binary stars
}

\section{Wilhelm Kley}

Institut für Astronomie \& Astrophysik and Kepler Center for Astro and Particle Physics, Universität Tübingen, Auf der Morgenstelle 10, D-72076 Tübingen, Germany email: wilhelm.kley@uni-tuebingen.de

\begin{abstract}
As of today more than 30 planetary systems have been discovered in binary stars. In all cases the configuration is circumstellar, where the planets orbit around one of the stars. The formation process of planets in binary stars is more difficult than around single stars due to the gravitational action of the companion. An overview of the research done in this field will be given. The dynamical influence that a secondary companion has on a circumstellar disk, and how this affects the planet formation process in this challenging environment will be summarized. Finally, new fully hydrodynamical simulations of protoplanets embedded in disks residing in a binary star will be presented. Applications with respect to the planet orbiting the primary in the system $\gamma$ Cephei will be presented.
\end{abstract}

Keywords. Planet formation, protoplanetary disk, binary stars, hydrodynamics

\section{Observational Data}

Planet formation is obviously a process that may occur around single as well as in multiple stars, which is indicated by the detection of about 35 planetary systems that reside in a binary or even multiple star configurations. All of the observed systems display a so called S-type configuration in which the planets orbit around one of the stars and the additional star, the companion or secondary star, acts as a perturber to this system. In this review we shall refer to the secondaries as single objects, even if they may be multiple. As indicated in Table 1 the distances of the secondaries to the host star of the planetary system range from very small values of about $20 \mathrm{AU}$ for Gl 86 and $\gamma \mathrm{Cep}$ to several thousand AU. As the influence of the secondaries on the planet formation process will obviously be smaller for larger distances, the mere existence of the last three systems, below the horizontal separation line in the table, represent a special challenge to any kind of planet formation process. Interestingly, all the additional systems have separations larger than $100 \mathrm{AU}$, and some several $1000 \mathrm{AU}$, but note that the list is very incomplete for larger separations. Despite the actual detection of planets in binary systems there is additional circumstantial evidence of debris disks (which are thought of as a byproduct of the planet formation process) in binary systems as indicated by recent Spitzer data. Here, for S-type configurations it is found that disks around an individual star of the binary exist mainly for binary separations larger than 50 AU, while P-type circumbinary debris disks are detected only in very tight binaries with $a_{b i n}$ smaller than about 3 AU (Trilling et al. 2007).

\section{Constraints on the planet formation process in binary stars}

In a binary star system the formation of planets is altered and effectively handicapped due to the dynamical action of the companion and the subsequent change in the internal structure of the protoplanetary disks. The tidal torques of the companion generate strong spiral arms in the circumstellar disk of the primary and angular momentum will be 


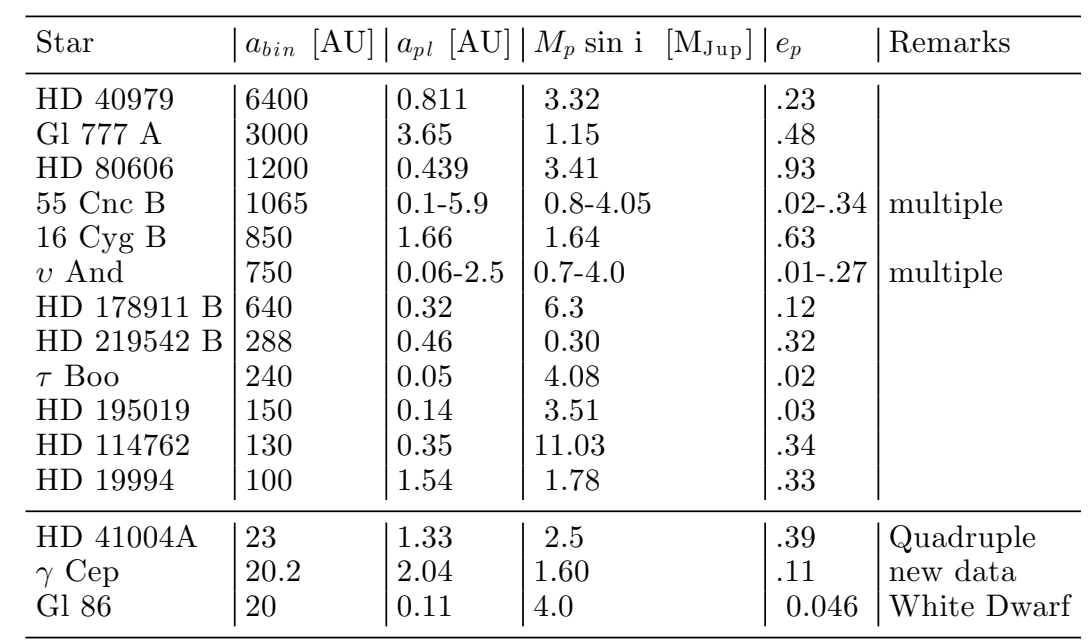

Table 1. A representative selection of planets in binary systems (see Eggenberger et al. 2004).

transferred to the binary orbit which in turn leads to a truncation and restructuring of the disk. Using analytical and numerical methods Artymowicz \& Lubow (1994) have shown how the truncation radius $r_{t}$ of the disk depends on the binary separation $a_{b i n}$, its eccentricity $e_{b i n}$, the mass ratio $q=M_{2} / M_{1}$ (where $M_{1}, M_{2}$ denote are the primary and secondary mass, respectively), and the viscosity $\nu$ of the disk. For typical values of $q \approx 0.5$ and $e_{b i n}=0.3$ the disk will be truncated to a radius of $r_{t} \approx 1 / 3 a_{b i n}$ for typical disk Reynolds numbers of $10^{5}$ (Artymowicz \& Lubow 1994, Armitage et al. 1999). For a given mass ratio $q$ and semi-major axis $a_{b i n}$ an increase in $e_{b i n}$ will reduce the size of the disk while a large $\nu$ will increase the disks radii.

Whether these changes in the disk structure have an influence on the likelihood of planet formation in such disks has been a matter of debate. The dynamical action of the secondary induces several consequences which are adverse to planet formation: $i$ ) it changes the stability properties of orbits around the primary, ii) it reduces the lifetime of the disk, and iii) it increases the temperature in the disk.

In a numerical study Nelson 2000 investigated the evolution of an equal mass binary with a $50 \mathrm{AU}$ separation and an eccentricity of 0.3 . He argued that both main scenarios of planet formation (i.e. through core instability or gravitational instability) are strongly handicapped, because the eccentric companion will induce a periodic heating of the disk up to temperatures possibly above $1200 \mathrm{~K}$. Since the condensation of particles as well as the occurrence of gravitational instability require lower temperatures, planet formation will be made more difficult. Clearly the strength of this effect will depend on the binary orbital elements, i.e. $a_{b i n}$ and $e_{b i n}$ and its mass ratio $q$.

However, recent numerical studies of the early planetesimal formation phase in rather close binaries with separations of only 20-30 AU show that it is indeed possible to form planetary embryos in such systems (Lissauer et al. 2004, Turrini et al. 2005, Quintana et al. 2007).

Already in ordinary planet formation around single stars the lifetime of the disk represents a limiting factor in the formation of planets from such a disk. It has been suspected that the dynamical action of a companion will limit the lifetime of disks substantially and place even tighter constraints on the possibility of planet formation. However, a recent analysis of the observational data of disks in binary stars finds no or very little change in 
the lifetimes of the disks at least for separations larger than about $20 \mathrm{AU}$ (Monin et al. 2007).

\section{Evolution of protoplanets in disks}

In this section we present models of the evolution and growth of planetary cores in a binary system where the orbital elements of the binary have been chosen to match the system $\gamma$ Cep quite closely. The data for $\gamma$ Cep have been taken from Hatzes et al. (2003) which do not include the most recent improvements by Neuhäuser et al. (2007). This system is the tightest binary system known to contain a Jupiter-sized protoplanet. For this reason, it has attracted much attention in past years. Several studies looked at the stability and/or the possibility of (additional) habitable planets in the system (e.g. Dvorak et al. 2004, Turrini et al. 2004, Haghighipour 2006, Verrier \& Evans 2006). Due to the enhanced influence of the secondary on the disk this system presents a strong challenge to any kind of planet formation process. Following other studies (Thébault et al. 2004), we assume that that the formation of planetesimals within the protoplanetary disk around the primary has been possible and start our investigation at the point where the planetary core has been grown to about $30 M_{\text {Earth }}$. From this point on we follow the orbital evolution and growth of the planetary core in the disk.

\subsection{Model definition}

For the present study we have chosen a binary with $M_{1}=1.59 M_{\odot}, M_{2}=0.38 M_{\odot}$, $a_{b i n}=18.5 \mathrm{AU}$ and $e_{b i n}=0.36$, which translates into a binary period of $P=56.7 \mathrm{yrs}$. The primary star is surrounded by a flat circumstellar disk, where the binary orbit and the disk all lie in one plane, i.e. they are coplanar. The typical dynamical timescale in the disk, the orbital period at a few AU, is substantially shorter than the binary period, but in a numerical simulation the system's evolution can only be followed on these short dynamical time scales. To simplify the simulations we assume that the disk is vertically thin and perform only $2 \mathrm{D}$ hydrodynamical simulations of an embedded planet in a circumstellar disk which is perturbed by the secondary. We assume that the effects of the intrinsic turbulence of the disk can be described approximately through the viscous Navier-Stokes equations, which are solved by a finite volume method which is second order in space and time. To substantiate our results we have utilized two different codes RH2D (Kley 1999, Kley 1989) and NIRVANA (Nelson et al. 2000, Ziegler \& Yorke 1997). The disk is assumed to be locally isothermal where the ratio of the vertical thickness $H$ to the distance $r$ from the primary is constant, here $H / r=0.05$. For the viscosity an $\alpha$ type parameterization is used with $\alpha=0.02$.

In the runs presented the computational domain covers a radial range from 0.5 to 8 $\mathrm{AU}$, and 0 to $2 \pi$ in azimuth. This is covered with an equidistant $300 \times 300$ grid. To allow for parameter studies we have found it highly useful to increase the performance of the code and have implemented the FARGO-algorithm to the code RH2D which is particularly designed to model differentially rotating flows (Masset 2000). For our chosen radial range and grid resolution we find a speed-up factor of about 7.5 over the standard case. Then, applying a Courant number of 0.75 still about 160,000 timesteps are required for only 10 binary orbits for our radial range and a standard resolution of $300 \times 300$.

\subsection{Disk equilibration and effects on the planetary orbit}

Before inserting the planet into the disk it is necessary to first relax the disk in the binary to its equilibrium configuration in the presence of the secondary. This makes sure that the calculated planetary evolution is not spoiled by long term transients due to 

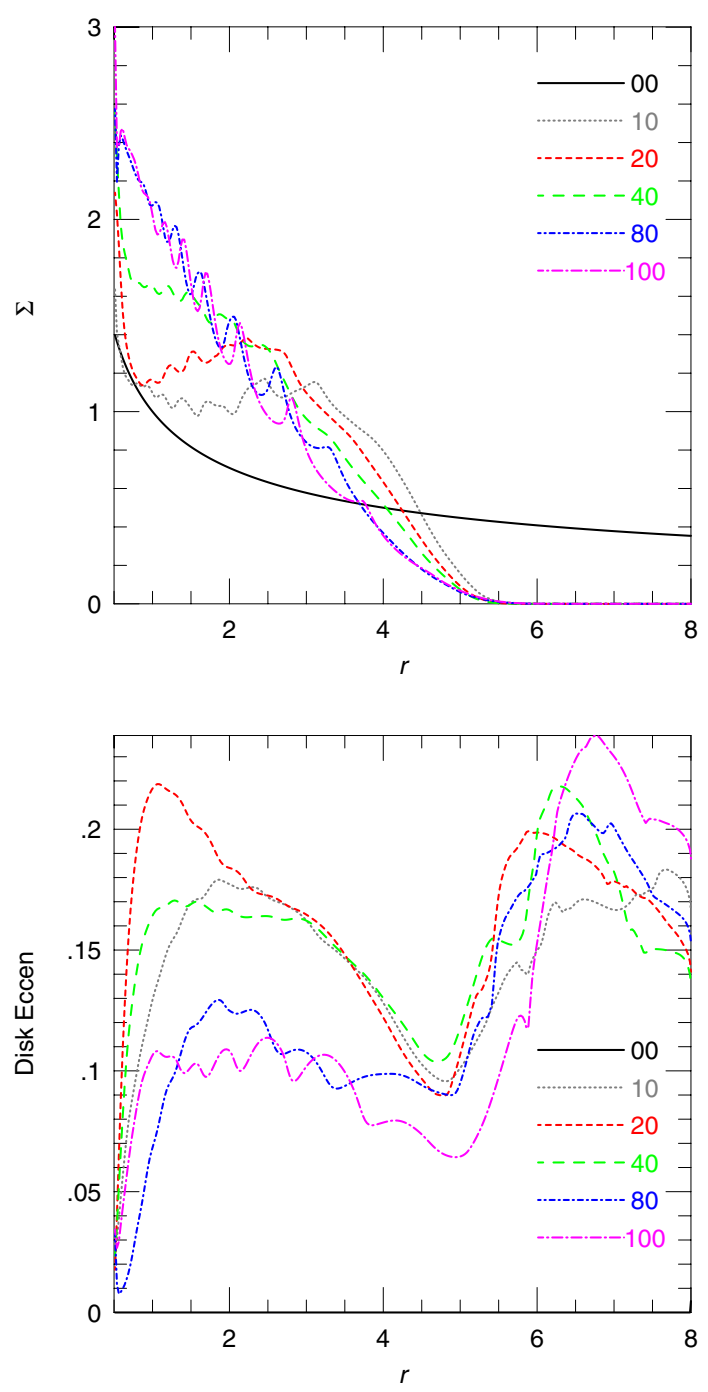

Figure 1. Azimuthally averaged disk structure at different evolutionary times given in binary orbits. On the left the surface density $\Sigma(r)$ is displayed and on the right the mean eccentricity $e(r)$.

the influence of the secondary star. In Fig. 1 we display such an initial settling of the disk with no planet. The initial surface density profile $\Sigma_{0}(r) \propto r^{-1 / 2}$ is given by the dark solid line, and the other lines represent the azimuthally averaged density during the subsequent evolution. As can be seen from the figure the disk is truncated very soon in the simulations (in fact after one or two orbits) and then re-adjusts as a whole on longer, viscous timescales to reach equilibrium at around 60-70 binary orbits. The disk is still perturbed periodically at each orbit due to the eccentric orbit of the secondary. At around each periastron strong spiral arms appear in the disk which are then damped until apoastron. However, the azimuthally averaged density structure at $t=80$ and $t=100$ does not differ anymore. During the process of equilibration the average eccentricity of the disk, $e_{d i s k}$, settles to a value of about 0.1 in the bulk part of the disk. Only in the 

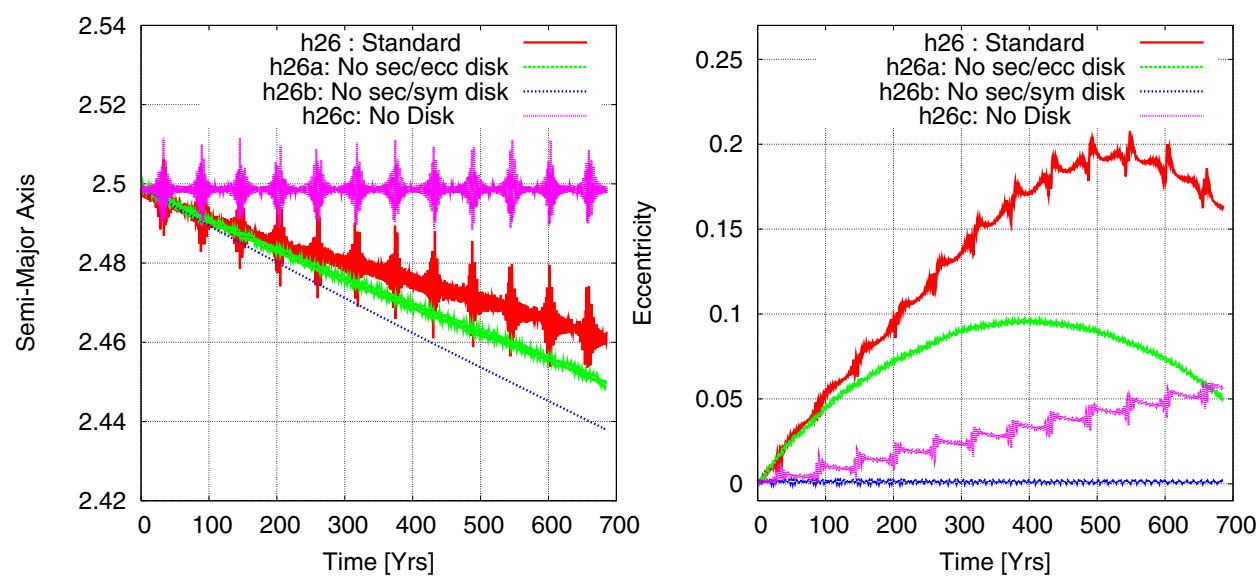

Figure 2. Evolution of the semi-major axis and eccentricity for fiducial models where either the disk or the binary have been switched off individually to test their influence separately.

outer low density parts of the disk the eccentricity remains high due to the disturbance of the secondary. This eccentric disk shows a coherent slow retrograde precession with a period of about 700 yrs or 14 binary orbits, see also Fig. 4 below.

The final two-dimensional density structure at the time $t=100$ is then used as the initial condition for the embedded planet simulations. The total mass of the disk is rescaled to $3 M_{J u p}$ and the planet of $30 M_{E a r t h}$ is placed on a circular orbit at a given semi-major axis (distance) from the primary star, ranging from 2.0AU to 3.5AU.

Before performing a full run where the planet may evolve and accrete material over a long time scale evolution we study a few test cases where individual effects are switched off selectively in order to estimates its influences. After inserting the protoplanet on a circular orbit at 2.5 AU we generally expect that, in addition to the typical planetdisk interaction, its orbital elements will change due to the gravitational influence of the binary and the distorted disk. To differentiate the different contributions we have decided to check the origin of the dynamical behavior, through a variation of physical conditions. The standard model resembles the true physical situation where the planet feels the full influence of the binary and the disk which is perturbed by the binary. In the other setups we switch the various contributions on and off. Not to add additional complications to the problem the protoplanet is not allowed to accrete here such that its mass remains fixed at $30 M_{E a r t h}$. The results are displayed in Fig. 2, where the semi-major axis and eccentricity of the planet are shown for four different setups. The standard model (h26, dark solid line) refers to the full model (including binary and disk) as just described, in model (h26a, dashed bright line) the mass of the secondary has been switched off to test its influence, and the planet evolves in the initially eccentric disk which becomes more circular during the evolution because of the lacking secondary. In the next model (26b, dark dotted line), in addition to having no secondary the density in the disk has been azimuthally symmetrized keeping the radial distribution intact. Hence, this model suits as a reference of what happens in the single star case. In the last model (26c, fine dotted line) the secondary is present but the disk mass has been reduced, such that effectively only a 3-body problem is solved. The curves with the periodic bumps in Fig. 2 refer to the cases $(26,26 \mathrm{c})$ including the secondary while the smoother curves describe the situation where the secondary star has been excluded (26a,26b). The bumps occur with the binary 
period and indicate the 'kick' the planet experiences due to the interaction with the spiral arms in the disk. The results show that the main contribution to the initial growth of planetary eccentricity $e_{p}$ is in fact the eccentric disk. The eccentricity $e_{p}$ for models (h26 and h26a) rise initially with the same speed but due to the fact that without the secondary star the disk slowly circularizes, such that the model (h26a) falls behind the full model (which reaches $e_{p}=0.2$ at $t \approx 500$ ) and then $e_{p}$ begins to drop off after a time of about $400 \mathrm{yrs}$ after insertion of the protoplanet. The maximum eccentricity reached for the reduced case (with no binary) is only $e_{p}=0.1$. In the symmetrized disk case without secondary (26b) the planet behaves as expected for single star disks, it displays inward migration (the fastest for all cases) and the eccentricity remains zero. The case with only the binary (h26c) does not show any change in the semi-major axis $a_{p}$ but rather a slow secular rise in $e_{p}$. This initially linear rise represents the first part of the long term periodic variation of $e_{p}$ induced by the binary which has a period of approximately $T_{\text {sec }}=4 / 3 M_{*} / M_{b i n}\left(a_{p} / a_{b i n}\right)^{-3} P_{p} \sim 2200 P_{p}$, where $P_{p}$ is the period of the planetary orbit. Additional test cases with similar setup but different starting distances $a_{p}=3.0$ and 3.5 AU display similar behavior. For the largest initial distance 3.5AU there are clear jumps in $e_{p}$ evolution visible at each individual periapse.
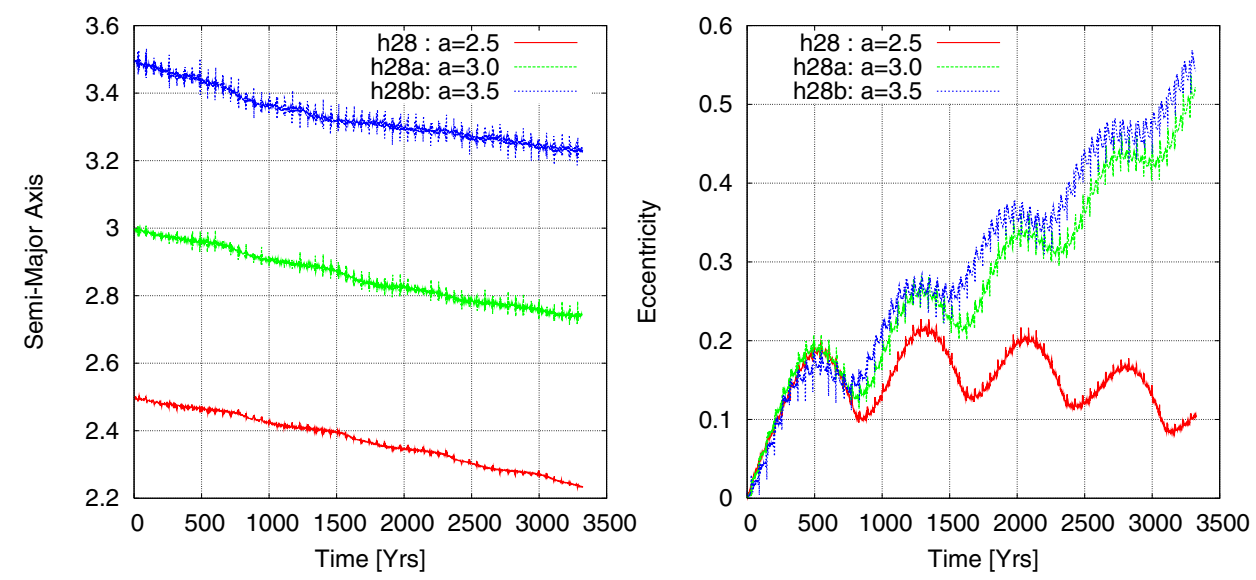

Figure 3. The evolution of the semi-major axis and eccentricity for planets released at different distances from the binary, i.e. 2.5, 3.0 and 3.5 AU.

\subsection{Evolving planets without mass accretion}

Planetary cores form in the outer cooler regions of protoplanetary disks beyond the so called ice-line. However, in a binary star system the outer disk is affected most by the secondary, and to find possible restrictions on the planet forming regions in the disk it is important to analyze the evolution of cores near the outer parts of the disk. To study the effect of initial position we start our embryos at 3 different locations in the disk 2.5, 3.0 and 3.5 AU always on a circular orbit, and choose again non-accreting cores. Because the initial characteristic growth time of the cores may be long, even in comparison to the orbital period of the binary these set of runs constitute a test suite to estimate the orbital evolution of protoplanets in the disk. The results for the semi-major axis and eccentricity evolution of the planet are displayed in Fig. 3, where the only difference in the three cases is the release distance $(2.5,3.0$ and $3.5 \mathrm{AU})$ of the planet. From all three locations the 


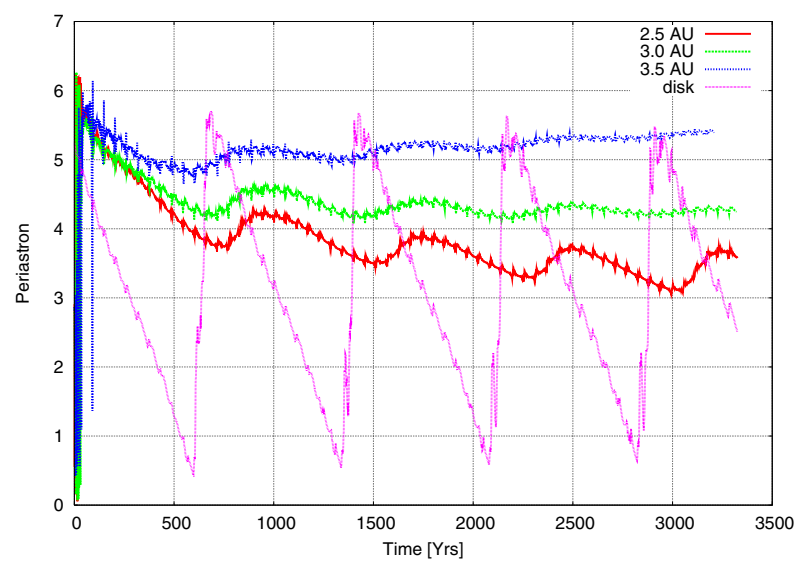

Figure 4. Time evolution of the argument of pericenter (periastron) for an embedded planet starting at 3 different locations and the protoplanetary disk.

planets migrate inwards at approximately the same rate with the tendency for a slow down for the two outer cases. However, the different initial starting radii lead to a very different eccentricity evolution. While only the innermost planet (starting at 2.5 AU) shows a finite eccentricity evolution the two outer cases display a very strong increase in their eccentricity beyond $e_{p}=0.5$ after about 55 binary orbits. Clearly the strongly disturbed disk in the outer regions at around $4 \mathrm{AU}$ significantly perturbs the orbits of the protoplanet and induces initially such high eccentricities that the resulting elongated orbits become more and more influenced by the action of the binary. This increases the eccentricities to such high values that the orbits will certainly be unstable eventually. The region of stability in this orbital domain has been analyzed through simple N-body simulations (e.g. Dvorak et al. 2004, Turrini et al. 2004) which match very well to our results here.

As the planets move on non-circular orbits in an eccentric disk and binary, a temporal change of the apsidal line may be expected. In Fig. 4 the evolution of the argument of pericenter of the planets and the disk are plotted versus time. The innermost planet has on average a periastron angle of about $200 \mathrm{deg}$ while the outer planets have a larger angle. For the binary the angle of periastron lies fixed at 0 degrees, and the disk is slowly precessing retrograde as pointed out above and indicated in Fig. 4 by the tightly dashed line. The influence of the disk on the planets is visible by the slight oscillations of the periastron angle about the mean with the same period as the oscillations in the eccentricity. The innermost planet has approximately a phase shift of $180 \mathrm{deg}$ with respect to the binary and is nearly in an anti-symmetric state while the other planets are lagging behind this configuration.

\subsection{Evolution with mass accretion}

To estimate the influence of accretion on the planetary evolution we have performed models where the mass of planets is allowed to grow due to accretion from the ambient disk. This accretion process is modelled by taking out mass within a given radius $r_{a c c}$ around the planet at a prescribed rate. Whenever the center of a gridcell is closer than about half of the planetary Hill radius, $r_{a c c}=1 / 2 R_{H i l l}$, the density in that cell is reduced by a factor $1-f_{a c c} * \Delta t$, where $\Delta t$ the the actual timestep of the computation and $f_{a c c}$ is a model dependent reduction factor. The rate is doubled whenever the distance is smaller 


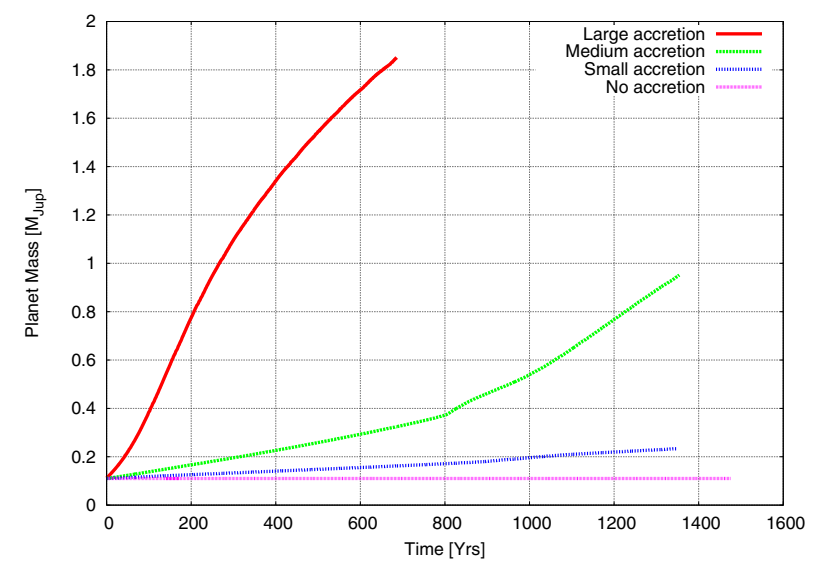

Figure 5. Mass growth of a protoplanet released at a distance of $2.5 \mathrm{AU}$ with an initial mass of about $30 M_{\text {Earth }}$ for different imposed accretion rates.
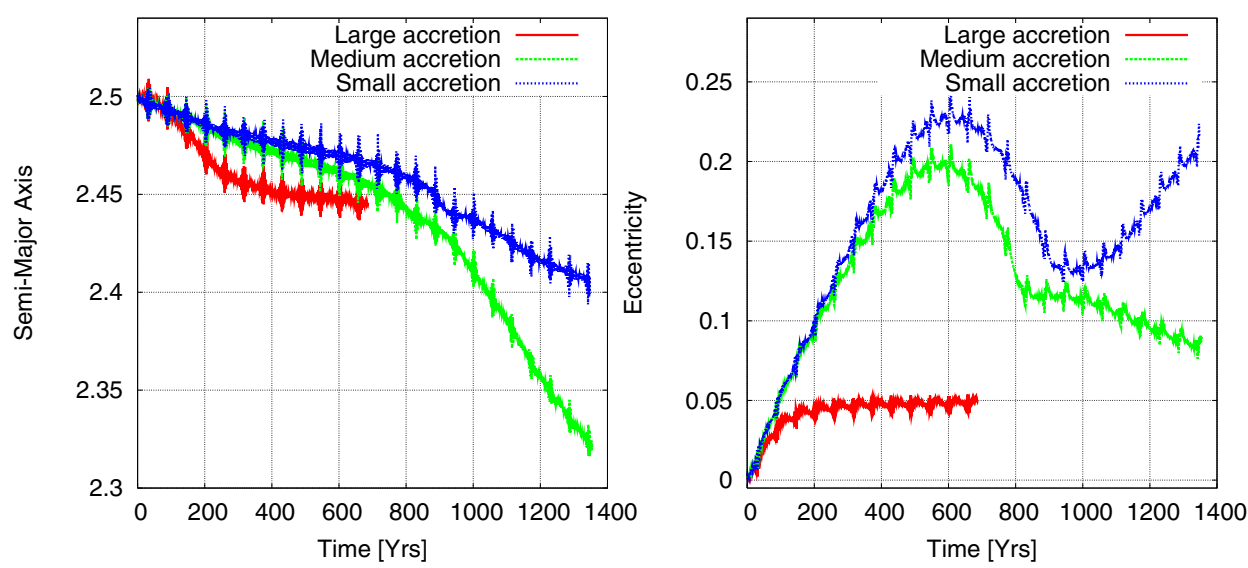

Figure 6. The evolution of the semi-major axis and eccentricity for planets released at an initial distance of 2.5 AU for different mass accretion rates as given in Fig. 5.

than 1/4 $r_{a c c}$ (for details see Kley 1999). In Fig. 5 we display the mass evolution of a planetary core released at a distance of $2.5 \mathrm{AU}$ for different accretion rates. The largest accretion rate refers to a value of $f_{a c c}=1.0$, and the medium and small to $10^{-1}$ and $3 \times 10^{-2}$, respectively. For the large accretion rate the mass of the planet increases very rapidly and reaches about $2 M_{J u p}$ after 800 yrs while the other models take accordingly longer.

The migration rate is initially similar for all accretion rates but then accelerates as the mass of the planet increases (left panel of Fig. 6), and finally it slows down because the mass reservoir of the disk becomes exhausted. For the same reason (faster reduction of disk material) the final eccentricity of the planet is smaller for higher accretion rates. Hence, the detailed evolution of the orbital elements of the planet depends on the rate of mass accretion onto the planet. The efficacy of the accretion process cannot be determined 
straightforwardly but is given for example by thermal processes in the vicinity of the growing planet. In our simulations we did not find a single case of outward or highly reduced migration in the case of smaller masses (accretion rates). However, the migration rate may also be affected by thermal processes in the disk. These will be studied in a future investigation.

\section{Summary}

In this contribution we have concentrated on the planetary formation process in the system $\gamma$ Cep which places, due the relatively tight binary star, severe constraints on the formation process. To study the effect of the binary we have followed the evolution of planetary embryos interacting with the ambient protoplanetary disk which is perturbed by the secondary star.

As suspected, the perturbations of the disk, in particular the periodic creation of strong tidally induced spiral density arms, lead to non-negligible effect on the planetary orbital elements. While embryos placed in the disk at different initial distances from the primary star continue to migrate inwards at approximately the same rate, the eccentricity evolution is markedly different for the individual cases. If the initial distance is beyond about $a \gtrsim 2.7 \mathrm{AU}$ the eccentricity of the embryo continues to rise to very high values and apparently only due to the damping action of the disk the orbit remains bound. The main excitation mechanism of the initial rise of the eccentricity is the perturbed disk and the spiral arms near the outer edge of the disk, and for larger $e_{p}$ the eccentric binary will pump it up to even larger values. This finding is consistent with the stability analysis of planetary orbits in $\gamma$ Cep in the case of pure 3-body simulations.

For a disk mass of $3 M_{J u p}$ a $1.6 M_{J u p}$ planet can easily be grown, and the final semimajor axis and eccentricity are also in the observed range of the $\gamma$ Cep planet for suitable accretion rates onto the planet. One of the major problems in forming a planet in this binary system via the core instability model is the problem of the formation of the planetary core in the first place. Due to the large relative velocities induced in a planetesimal disk especially for objects of different sizes the growth process is also problematic in itself, see however new results by Xie \& Zhou (2008) in this volume.

Hence, the formation of the Jupiter sized planet observed in $\gamma$ Cep via the standard scenarios remains difficult but may not be impossible. Future research will have to concentrate on additional physical effects such as radiative transport, three-dimensional effects and self-gravity of the disk.

\section{Acknowledgements}

This review is based on joint work with Richard Nelson from London (GB).

\section{References}

Armitage, P. J., Clarke, C. J., \& Tout, C. A. 1999, MNRAS, 304, 425

Artymowicz, P. \& Lubow, S. H. 1994, ApJ, 421, 651

Dvorak, R., Pilat-Lohinger, E., Bois, E., et al. 2004, in Revista Mexicana de Astronomia y Astrofisica Conference Series, Vol. 21, ed. C. Allen \& C. Scarfe, 222-226

Eggenberger, A., Udry, S., \& Mayor, M. 2004, A\& $A, 417,353$

Haghighipour, N. 2006, ApJ, 644, 543

Hatzes, A. P., Cochran, W. D., Endl, M., et al. 2003, ApJ, 599, 1383

Kley, W. 1989, A\&\&A, 208, 98

Kley, W. 1999, MNRAS, 303, 696 
Lissauer, J. J., Quintana, E. V., Chambers, J. E., Duncan, M. J., \& Adams, F. C. 2004, in Revista Mexicana de Astronomia y Astrofisica Conference Series, 99-103

Masset, F. 2000, A\& $A S, 141,165$

Monin, J.-L., Clarke, C. J., Prato, L., \& McCabe, C. 2007, in Protostars and Planets V, ed. B. Reipurth, D. Jewitt, \& K. Keil, 395-409

Nelson, A. F. 2000, ApJ, 537, L65

Nelson, R. P., Papaloizou, J. C. B., Masset, F. S., \& Kley, W. 2000, MNRAS, 318, 18

Neuhäuser, R., Mugrauer, M., Fukagawa, M., Torres, G., \& Schmidt, T. 2007, A\&A, 462, 777

Quintana, E. V., Adams, F. C., Lissauer, J. J., \& Chambers, J. E. 2007, ApJ, 660, 807

Thébault, P., Marzari, F., Scholl, H., Turrini, D., \& Barbieri, M. 2004, A\&A, 427, 1097

Trilling, D. E., Bryden, G., Beichman, C. A., et al. 2007, ArXiv Astrophysics e-prints

Turrini, D., Barbieri, M., Marzari, F., Thebault, P., \& Tricarico, P. 2005, Memorie della Societa Astronomica Italiana Supplement, 6, 172

Turrini, D., Barbieri, M., Marzari, F., \& Tricarico, P. 2004, Memorie della Societa Astronomica Italiana Supplement, 5, 127

Verrier, P. E. \& Evans, N. W. 2006, MNRAS, 368, 1599

Xie, J. -W. \& Zhou, J. -L. 2008, in: Y.-S. Sun, S. Ferraz-Mello \& J.-L. Zhou, (eds.),IAU symposium 249: Exoplanets: Detection, Formation and Dynamics, Proc. IAU Symposium No. 249 (Suzhou,China), p. 419

Ziegler, U. \& Yorke, H. 1997, Computer Physics Communications, 101, 54 